\title{
High-intensity-exercise-induced intestinal damage is protected by fermented milk supplemented with whey protein, probiotic and pomegranate (Punica granatum L.)
}

\author{
Fernanda M. Chaves $^{1}$, Igor L. Baptista ${ }^{1}$, Fernando M. Simabuco ${ }^{1}$, Paula G. F. Quaresma ${ }^{2}$, \\ Fabiola L. Pena ${ }^{1}$, Rosangela M. N. Bezerra ${ }^{1}$, Jose R. Pauli ${ }^{1}$, Diogo T. da Cunha ${ }^{1}$, \\ Patricia L. Campos-Ferraz ${ }^{1}$ and Adriane E. C. Antunes ${ }^{1 *}$ \\ ${ }^{1}$ School of Applied Sciences, University of Campinas (FCA/UNICAMP), Rua Pedro Zaccaria, n. 1300, 13484-350, Limeira, Brazil \\ ${ }^{2}$ School of Medical Sciences, University of Campinas (FCM/UNICAMP), Rua Tessalia Vieira de Camargo, n. 126, 13083-887, \\ Campinas, Brazil
}

(Submitted 13 October 2017 - Final revision received 17 January 2018 - Accepted 5 February 2018)

\begin{abstract}
Here we evaluated the effect of fermented milk supplemented with whey protein (approximately $80 \%$ protein), probiotic (Bifidobacterium animalis subsp. lactis BB12) and pomegranate juice (Punica granatum L.) on the physical performance, intestinal motility and villi structure, inflammatory markers and intestinal microbiota of rats under high-intensity acute exercise. In all, twenty-four Wistar rats were separated into groups: control (Ctrl), supplemented (Supp), exercised (Exe) and exercised and supplemented (Exe+Supp). Rats in the Supp groups received fermented milk during 6 weeks by oral administration. At the end of the supplementation period, the Exe groups were submitted to high-intensity acute exercise on a treadmill. We found that intense acute exercise caused changes in the intestinal villi interspace, changes in the proportion of Lactobacillus species and an increase in Clostridium species, as well as a decrease in intestinal motility. Supplementation increased intestinal motility, and maintained the intestinal villi interspace and the natural microbiota proportions of the exercised rats. Physical performance was not improved by fermented milk supplementation. We conclude that the fermented milk containing whey protein, B. animalis (BB12) and pomegranate juice can re-establish intestinal microbiota and protect the animals from the undesirable effects of intense acute exercise.
\end{abstract}

Key words: Exercise: Probiotics: Phenolics: Microbiota

Whey protein provides amino acids that are used mainly during the synthesis of glutamine in muscles: branched-chain amino acids $(26 \%)$ and glutamate $(6 \%)^{(1)}$. Therefore, these amino acids can be used to preserve muscular glutamine stock, which is widely required by immune system cells as an energy source in clinical states ${ }^{(2,3)}$ and during exercise ${ }^{(4,5)}$. There is evidence that glutamine has an important role in the maintenance of intestinal barrier function ${ }^{(6)}$, which is also negatively regulated by prolonged and intense physical exercise, reducing intestinal absorption capacity ${ }^{(7)}$.

Physical exercise alters the immune system and cell function $^{(7)}$. Therefore, intense exercise can increase the incidence of infections in the gastrointestinal tract and upper respiratory tract $^{(8)}$. These physiological changes are related to modifications of structural proteins known as tight junctions. These proteins remodel because of constant changes in blood flow in the intestinal region ${ }^{(9)}$. The joint action of epithelial cells and lymphoid tissue associated with the intestine and luminal bacteria can prevent pathogen invasion, thereby exerting an important barrier function ${ }^{(10)}$. Transmembrane proteins have been identified on tight junctions, where they regulate the immune system, water and solute paracellular permeability ${ }^{(11,12)}$. Two of these proteins have been extensively studied in animal models: zonula occludens-1 (ZO-1), a cellular adhesion protein responsible for signal transduction in cell-cell junctions, and claudin-1, an integral membrane protein that forms a paracellular barrier, playing an important role regulating epithelial cell permeability ${ }^{(12,13)}$.

Probiotic supplementation of athletes can help reduce the frequency, severity and duration of gastrointestinal problems, as well as of respiratory illness. This occurs probably because of the direct interaction between these cultures, intestinal microbiota and the immune system ${ }^{(14)}$.

Furthermore, intense or strenuous exercise can lead to an imbalance of reactive oxygen species production and the endogenous antioxidant system, resulting in oxidative stress ${ }^{(15)}$. Multiple studies have reported an antioxidant and antiinflammatory action for pomegranate (Punica granatum, L.),

Abbreviations: Ctrl, control; Exe, exercised; Exe + Supp, exercised and supplemented; ILT, incremental load test; Supp, supplemented; ZO-1, zonula occludens-1. 
which is associated with the presence of phenolic compounds, mainly gallic acid ${ }^{(16-18)}$

Therefore, here we aimed to evaluate the effect of supplementation with fermented milk containing whey protein, probiotic and pomegranate juice on the physical performance, intestinal microbiota and motility, intestinal villi structure and inflammatory markers of rats submitted to high-intensity acute exercise.

\section{Methods}

\section{Fermented milk production}

The fermented milk was composed of skimmed powder milk $\left(\right.$ Molico $\left.^{\circledR}\right)$, concentrated whey protein (Alibra ${ }^{\circledR}$, approximately $80 \%$ de protein), maltodextrin $\left(\right.$ SweetMix $^{\circledR}$ ), fructose (Lowçucar $^{\circledR}$ ), pomegranate juice (var. Wonderful, obtained by fruit processing with industrial fruit pulper, model DPT-75; Tomasi $^{\circledR}$ ), potassium sorbate $\left(\right.$ SweetMix $\left.^{\circledR}\right)$, starter culture Streptococcus thermophilus (TA 072) and probiotic Bifidobacterium animalis subsp. lactis (BB12), kindly provided by Danisco and Chr-Hansen, respectively. The fermentation was carried out at $42^{\circ} \mathrm{C}$ on Biochemical Oxygen Demand (model TE-371; Tecnal $\left.{ }^{\circledR}\right)$ until pH $4 \cdot 6 \pm 0 \cdot 1$.

\section{Protein content}

The protein content of the fermented milk was determined by the Micro-Kjeldahl method, applying a conversion factor of $6 \cdot 38^{(19)}$.

\section{Probiotic viability on the fermented milk}

The viability of $B$. animalis subsp. lactis (BB12) was evaluated in the fermented milk at 0,14 and $28 \mathrm{~d}$ after production, using TOS-MUP media and incubation under anaerobiosis (AnaeroGen; Oxoid ${ }^{\circledR}$ ) at $37^{\circ} \mathrm{C}$ for $72 \mathrm{~h}$.

\section{Phenolic content and antioxidant activity of pomegranate juice and blood serum}

Determination of total phenolic content from pomegranate juice was performed using the Folin-Ciocalteau method, as described by Musci \& Yao ${ }^{(20)}$

The ferric-reducing antioxidant power (FRAP) method was used to evaluate the antioxidant activity. In brief, samples of pomegranate juice extract were placed in a ninety-six-well plate: $20 \mu \mathrm{l}$ of sample, standard or water (blank); $30 \mu \mathrm{l}$ of distilled water; and $200 \mu \mathrm{l}$ of FRAP reagent, according to the method described by Benzie \& Strain ${ }^{(21)}$. Next, the plate was agitated and incubated at $37^{\circ} \mathrm{C}$ for $8 \mathrm{~min}$, and the absorbance was read at $595 \mathrm{~nm}$ using a spectrophotometer (BioTek, $\mathrm{EPOCH} / 2$ microplate reader with Gen5.3.03 software version). The antioxidant activity of rat blood serum was also evaluated using the FRAP method. Animal blood was collected and centrifuged at $2000 \boldsymbol{g}$ for $10 \mathrm{~min}$ (centrifuge model K241R; Centurion Scientific ${ }^{\circledR}$ ), and the supernatant was taken for analysis.

\section{Ethics declaration}

This work was approved by the Animal Use Ethics Committee (CEUA) of University of Campinas (protocol 4150-1) and was conducted under the ethics standards for animal experimentation of the Brazilian College of Animal Experimentation (COBEA).

\section{Experimental groups}

In all, twenty-four 10-week-old male Wistar rats weighing approximately $350 \mathrm{~g}$ (at the beginning of the experiment) were maintained in the Central Biotery from the School of Applied Sciences (FCA-UNICAMP). The rats were kept in polyethene cages, four animals per unit, at a controlled temperature of $25^{\circ} \mathrm{C}$ and $12 \mathrm{~h}$ light $-12 \mathrm{~h}$ dark cycles, receiving potable water and standard commercial diet $\left(\mathrm{Nuvilab}^{\circledR}\right)$, ad libitum.

Rats were distributed among four groups: (i) the control (Ctrl) group, without supplementation and with no exercise; (ii) the supplemented group (Supp), with supplementation and with no exercise; (iii) the only exercised (Exe) group, without supplementation but exercised on a treadmill; and (iv) the supplemented plus exercised group (Exe + Supp), supplemented with the fermented milk and exercised on a treadmill.

During the experimental period, the weights of the animals were measured weekly using stabilised semi-analytical scales (model UX620H; Marte Científica ${ }^{\circledR}$ ). A period of $12 \mathrm{~h}$ after an acute exercise session, the rats were anaesthetised with an intraperitoneal injection containing a mixture of ketamine hydrochloride and diazepam $(70: 30 ; 50 \mathrm{mg} / \mathrm{kg})$ and then euthanised. Colon and faeces samples were immediately frozen and stored at $-80^{\circ} \mathrm{C}$. Distal colon samples were frozen in isopentane cooled in liquid $\mathrm{N}_{2}$ for further histological, immunofluorescence and PCR analysis. Blood samples were collected to obtain serum.

\section{Supplementation with the fermented milk}

The composition of the fermented milk was calculated on the basis of the appropriate amount of whey protein $(20 \mathrm{~g} / \mathrm{d}$ of protein), as proposed by Witard et al. ${ }^{(22)}$. Each animal received $2 \mathrm{ml}$ of fermented milk, $5 \mathrm{~d}$ /week, containing 8.9 (sD 0.3) log colony-forming units (CFU) of B. animalis BB12, whey protein (calculated according to animal average weight, measured weekly) and pomegranate juice with a concentration of $2.625 \mathrm{mmol}$ of total polyphenols.

\section{Acute exercise protocol}

The rats were previously adapted on a rodent-motorised treadmill with electric shock stimulation (AVS Projetos ${ }^{\circledR}$ ) for 1 week (for $10 \mathrm{~min} / \mathrm{d}$ at $3 \mathrm{~m} / \mathrm{min}$ ). Then, rats performed the incremental load test (ILT) with an initial intensity of $6 \mathrm{~m} / \mathrm{min}$ at $0 \%$ with increasing increments of $3 \mathrm{~m} / \mathrm{min}$ every $3 \mathrm{~min}$ until exhaustion (i.e. maximum speed), which was defined as the time that rats touched the end of the treadmill five times in 1 min $^{(23)}$. The ILT was performed three times with an interval of $48 \mathrm{~h}$ between them. From the animals' physical performance in these tests, we randomly selected twelve rats to compose the Exe groups.

After this selection, rats were submitted to a 6-week treatment, which included supplementation with fermented milk or potable water (control), administered orally. On the 5th week of 
treatment, the Exe groups were again submitted to adaptation on the treadmill for 1 week. Then, the rats performed the ILT two times, with $48 \mathrm{~h}$ of resting between the tests. The ILT was conducted to establish performance data, such as maximum speed, time and distance. As no discrepancy was found between tests, we adopted the average values. After this period, the rats from the Exe groups were submitted to an acute aerobic exercise session with a continuous intensity corresponding to $85 \%$ of the maximum speed obtained in the ILT.

A period of $12 \mathrm{~h}$ after acute exercise, the animals were euthanised. The no-exercise groups were placed on the treadmill (turned off) for $15 \mathrm{~min}$ to mimic the stress caused by the ambience of a different cage. These animals were also euthanised after $12 \mathrm{~h}$. Our previous experience (undisclosed data) is that after $12 \mathrm{~h}$ of the exhaustive exercise session there may be increased inflammatory process and changes in the gut structure in rats. For this reason, we chose to perform euthanasia after $12 \mathrm{~h}$ of the exhaustive exercise session.

\section{RNA extraction and real-time PCR (quantitative $P C R$ )}

Extracted RNA ( $1 \mu \mathrm{g})$ was used for reverse transcription containing OligodT $(500 \mu \mathrm{g} / \mathrm{ml}), 10 \mathrm{~nm}$ dNTP Mix, $5 \times$ First Strand Buffer, $0.1 \mathrm{~m}$ dithiothreitol and $200 \mathrm{U}$ RT (MMLV Reverse Transcriptase; Promega ${ }^{\circledR}$ ). Reverse transcription was conducted at $70^{\circ} \mathrm{C}$ for $10 \mathrm{~min}$. The cycle continued at $42^{\circ} \mathrm{C}$ for $60 \mathrm{~min}$ after the addition of the enzyme. The enzyme was deactivated at $95^{\circ} \mathrm{C}$ for $10 \mathrm{~min}$.

Gene expression levels were assessed by fluorescence quantification with a QuantStudio Real-Time System (Thermo Fisher Scientific ${ }^{\circledR}$ ). The reaction consisted of complementary DNA diluted in a mix containing Power SYBR Green PCR Master Mix (Applied Biosystems ${ }^{\circledR}$ ), Milli-Q water and respective sense and antisense initiators. The cycling conditions were $60^{\circ} \mathrm{C}$ for $1 \mathrm{~min}, 50^{\circ} \mathrm{C}$ for $2 \mathrm{~min}$ and $95^{\circ} \mathrm{C}$ for $10 \mathrm{~min}$, and amplification occurred in forty cycles of denaturation at $95^{\circ} \mathrm{C}$ for $15 \mathrm{~s}$ with an annealing/extension step at $60^{\circ} \mathrm{C}$ for the $60 \mathrm{~s}$. Analyses were performed using the $2^{-\Delta \Delta C_{t}}$ method, where $\Delta \Delta C_{t}=\left(C_{t}\right.$ sample$C_{t}$ internal control from the same sample $)-\left(C_{t}\right.$ control $-C_{t}$ internal control from the same control sample). This formula is based on the supposition that the rate of $C_{t}$ change $v$. the rate of target copy change is identical for the gene of interest and the $\beta$-actin internal control.

All primer pairs were synthesised by Sigma Life Science, as follows: ACTB, 5'-GTCTCACCACTGGCATTGTG-3' and 5'-TCT CAGCTGTGGTGGT-3'; CLDN1， 5'-CTGGGAGGTGCCCTACT TT-3' and 5'-CCGCTGTCACACGTAGTCTT-3'; ZO-1, 5'-GAGGC TTCAGAACGAGGCTATTT-3' and 5'-CATGTCGGAGAGTAGA GGTTCGA-3'; IL1B, 5'-CACCTCTCAAGCAGAGCACAG-3' and 5'-GGGTTCCATGGTGAAGTCAAC-3'; IL6, 5'-TCCTACCCCAAC TTCCAATGCTC-3' and 5'-TTGGATGGTCTTGGTCCTTAGCC-3'; and TNF, 5'-AAATGGGCTCCCTCTCATCAGTTC-3' and 5'-TCT GCTTGGTGGTTTGCTACGAC-3'.

\section{Histology}

The intestinal structure of animals was analysed by histology. The distal colon was frozen in isopentane, cooled in liquid $\mathrm{N}_{2}$ and stored at $-80^{\circ} \mathrm{C}$, and then cut into portions of $10 \mu \mathrm{m}$ (thickness) using a cryostat (model CM1860; Leica ${ }^{\circledR}$ ). To reveal the general morphology, the non-fixed histological cuts were dyed in a solution of haematoxylin and eosin $(1 \% \mathrm{w} / \mathrm{h}$ for each). The images were obtained under a microscope (Leica ${ }^{\circledR}$ ) using the LAS $\mathrm{X}$ software.

From the obtained images, we assessed the distance between intestinal villi using ImageJ software. All four images were analysed for each rat, with three per experimentation group.

\section{Immunofluorescence}

Transversal sections from the distal colon for immunostaining were fixed in cooled acetone $\left(-20^{\circ} \mathrm{C}\right)$ with $0 \cdot 2 \mathrm{M}$ phosphate buffer for $10 \mathrm{~min}$, washed with saline PBS three times for $3 \mathrm{~min}$ each and then blocked with $0 \cdot 1$ glycine/ $0 \cdot 2 \%$ TritonX-100 in PBS buffer for $1 \mathrm{~h}$. Posteriorly, slides were incubated in a solution containing a primary antibody, $3 \%$ normal goat serum and $0.3 \%$ Triton $\mathrm{X}-100 / 0 \cdot 1 \mathrm{~m}$ (three times for $10 \mathrm{~min}$ each), assembled with Vectashield mounting medium for immunofluorescence with 40,6-diamididino-2-phenylindole (catalogue no. H-1200; Vector Labs $^{\circledR}$ ) and coverslips. The primary antibodies used for immunolocalisation were claudin-1 (code: 374900; Life Technologies do Brasil ${ }^{\circledR}$ ) and ZO-1/TJP1 (code: 402200; Life Technologies do Brasil ${ }^{\circledR}$ ). The secondary antibodies were donkey Cy3 anti-mouse (1:500; catalogue no. 715-165-150; Jackson Lab ${ }^{\circledR}$ ) and donkey Cy3 anti-rabbit (1:500; catalogue no. 711-165-152; Jackson Lab $^{\circledR}$ ). The images were obtained using a microscope and LAS X software.

\section{Myeloperoxidase activity}

The migration of neutrophils to rats intestines was evaluated through kinect-colourimetric assay of myeloperoxidase (MPO). Approximately $0 \cdot 3 \mathrm{~g}$ of distal colon was weighed and samples were homogenised in buffer $\left(0 \cdot 1 \mathrm{M} \mathrm{NaCl}, 0.02 \mathrm{M} \mathrm{NaPO}_{4}, 1.015 \mathrm{M}\right.$ $\mathrm{Na}$ EDTA, $\mathrm{pH} 4.7$ ) and centrifuged at $1509 \boldsymbol{g}$ for $15 \mathrm{~min}$. The sediment was submitted to hypotonic lysis $(1.5 \mathrm{ml}$ of $\mathrm{NaCl}$ solution at $0 \cdot 2 \%$, followed by the addition of an equal volume of $\mathrm{NaCl}$ solution at $1.6 \%$ and glucose $5 \%$ ). After centrifugation, the sediment was resuspended in $0.05 \mathrm{M} \mathrm{NaPO}_{4}$ buffer $(\mathrm{pH} 5.4$ ) containing $0.5 \%$ hexadecyltrimethylammonium bromide. After this step, the sediment was instantly frozen in liquid $\mathrm{N}_{2}$ three times, centrifuged at $11963 \boldsymbol{g}$ for $15 \mathrm{~min}$ and then again homogenised. The MPO activity in resuspended sediment was analysed by measuring alterations in optical density at $450 \mathrm{~nm}$, using tetramethylbenzidine (1.6 nM) and $\mathrm{H}_{2} \mathrm{O}_{2}(0.5 \mathrm{~mm})$. Results were calculated by comparing the optical density of the supernatant with a standard curve of neutrophil numbers (>95\% of purity).

\section{Microbiota analysis using next-generation sequencing}

The intestinal microbiome analysis was performed by NextGeneration Sequencing (NGS), by Neoprospecta ${ }^{\circledR}$. First, the sequencing library preparation was carried out in a two-step PCR protocol using $1 \mathrm{ng}$ of DNA. In the first PCR reaction, the V3-V4 primers, 314F (CCTACGGGRSGCAGCAG) and 806R (GGACTACHVGGGTWTCTAAT), were used at a concentration of $0.2 \mu \mathrm{M}^{(24,25)}$. This pair of primers has great taxonomy coverage in bacteria and archaea, recognising the highly variable 
regions V3 and V4 of the bacterial rRNA $16 \mathrm{~S}^{(26)}$. This first PCR primer contains the Illumina sequences based on TruSeq structure adapter (Illumina ${ }^{\circledR}$ ), allowing the second PCR with indexing sequences. The PCR reactions were always carried out in triplicate using Platinum Taq (Invitrogen ${ }^{\circledR}$ ). The final PCR reaction was cleaned up using AMPureXP beads (Beckman Coulter $^{\circledR}$ ) and samples were pooled in the sequencing libraries for quantitative PCR quantification using he KAPA Library Quantification Kit for Illumina platforms (KAPA Biosystems ${ }^{\circledR}$ ).

The libraries were sequenced using a MiSeq system, using the standard Illumina primers provided in the kit. After sequencing, a bioinformatics pipeline performs sequence demultiplexing, adaptor and primer trimming. To increase the reliability of the read, excluding possible diversity generated by chimeric amplicons or erroneous nucleotide incorporated in PCR, 100\% identical reads were clustered. If any cluster was represented by fewer than five reads, it was not considered in further analysis. Clustered sequences were then subjected to taxonomic classification comparing them with a $16 \mathrm{~S}$ rRNA database (NeoRefdb; Neoprospecta Microbiome Technologies ${ }^{\circledR}$ ). Sequences with at least $99 \%$ identity in the reference database were taxonomically assigned.

\section{Statistical analysis}

Sample size was calculated using free sample size calculating software G*Power version 3.1.9.2 (Franz, Universitat Kiel).

The sample was calculated considering the results observed by Chen et $a l .{ }^{(27)}$ and the variable swimming time (ST) in three groups of trained mice: vehicle (ST=4.8); LP10-1× (ST=9.0); and LP10-5 $\times(S T=23 \cdot 3)$. Considering these values, we have an effect size of 7.91 . With a power of $95 \%, 0.05$ level of statistical significance and effect size of 7.91 , the sample size for each group was calculated to be 2 . Considering that the effect of the base study was large, we decided to be conservative and increase the sample number to a total of twenty-four mice randomly assigned into one of the four experimental groups.

Values for initial weight $v$. final weight and weight curve were expressed as the means and standard deviation and assessed by two-way ANOVA and Bonferroni's post hoc test, using repeated measures. For the two independent group comparison, we used the Student's $t$ test. For microbiota assessment, we elaborate a ratio between order quantification ( $n$ Lactobacillale/ $n$ Clostridiales) and between genus ( $n$ Lactobacillus/ $n$ Clostridium). The ratio between the groups and values for Claudin-1, ZO-1, IL1B, IL-6 and TNFa were compared by one-way ANOVA with the Games-Howell post hoc test. The tests were chosen because the group ratios had unequal variances ( $n$ reduced by group). In this case, the Games-Howell test avoids error distortions familywise type I and $\alpha$-type $\mathrm{I}^{(28)}$. These statistical analyses were performed using GraphPad Prism 5 and SPSS 15.0.1 software. The significance considered for all tests was $P<0.05$.

\section{Results}

\section{Fermented milk}

Before the fermented milk preparation, we evaluated the phenolic content of pomegranate juice extracted directly from the fruit. In addition, we analysed the protein content of the whey protein concentrate. Juice obtained from pomegranate contained $4.64 \mathrm{mmol}$ polyphenols, and from this value we calculated its addition percentage to the fermented milk, aiming to reach $2.625 \mathrm{mmol}$ of polyphenols in the drink used for supplementation. The protein content of whey protein was $77 \cdot 3$ (sD 0.14 ) $\%$, and we calculated its addition percentage to reach an equivalent dose of $20 \mathrm{~g} / \mathrm{d}$ for the drink, corrected by animal average weight at each week. Also, we evaluated probiotic viability on the fermented milk, and we observed counts above $8.5 \log \mathrm{CFU} / \mathrm{ml}$ during all the product shelf life. The International Scientific Association for Probiotics and Prebiotics elaborated an Expert Consensus Document in which there is no minimal count of viable probiotic cells to include the claim of a probiotic food ${ }^{(29)}$. Instead, the recommendation is to prove the viability at the appropriate level used in supporting human studies, according to Bertazzoni et al. ${ }^{(30)}$ and according to the legislation for probiotics in Brazil ${ }^{(31)}$. However, some countries suggest $9 \log$ CFU per serving, such as Italy and Canada ${ }^{(29)}$. The proposed serving of the fermented milk intended for human consumption is $140 \mathrm{ml}$, which corresponds to $10.6 \mathrm{log}$ CFU of probiotic cells. Comparing this quantity of probiotic cells given to a $70-\mathrm{kg}$ human, it is equivalent to the quantity of probiotics given to the rats (approximately $500 \mathrm{~g}$ ) in this research. The supplementation with fermented milk did not result in adverse events in the rats.

\section{Animals' physical performance}

Fig. 1(a) shows results of the progressive test until exhaustion before treatment, showing that Exe and Exe + Supp groups presented similar performances. Fig. 1(b) shows results of the same test after treatment, showing no significant difference between groups. Fig. 1(c) presents results of acute exercise at $85 \%$ maximum capacity obtained in the progressive test (Fig. 1(b)) where no improvement in performance was observed after treatment with the fermented milk in comparison with the Exe-only group.

\section{Body evaluation and intestinal content}

The supplementation with fermented milk does not lead to weight gain in comparison with control rats (Fig. 2(a) and (b)). We highlighted that only supplemented animals presented less faecal gut content in comparison with the other groups, with no statistical difference (Fig. 2(c)).

\section{Histology and intestinal villi distance}

Fig. 3(A) presents distal colon histology, showing that the Supp group presented similar intestinal villi in comparison with the Ctrl group, whereas the Exe group presented larger villi interspace. The Supp + Exe group demonstrated preservation of the original interspace. Evaluating the distance between intestinal villi using ImageJ (Fig. 3(B)), we found that the Exe group presented significantly more space compared with the Ctrl and Supp group, indicating that high-intensity acute exercise can cause structural changes in this mucosa (one-way ANOVA: $P=0.001, \mathrm{a}=P=0.025 v$. Ctrl, $\mathrm{b}=P=0.098 v$. Supp). On the 

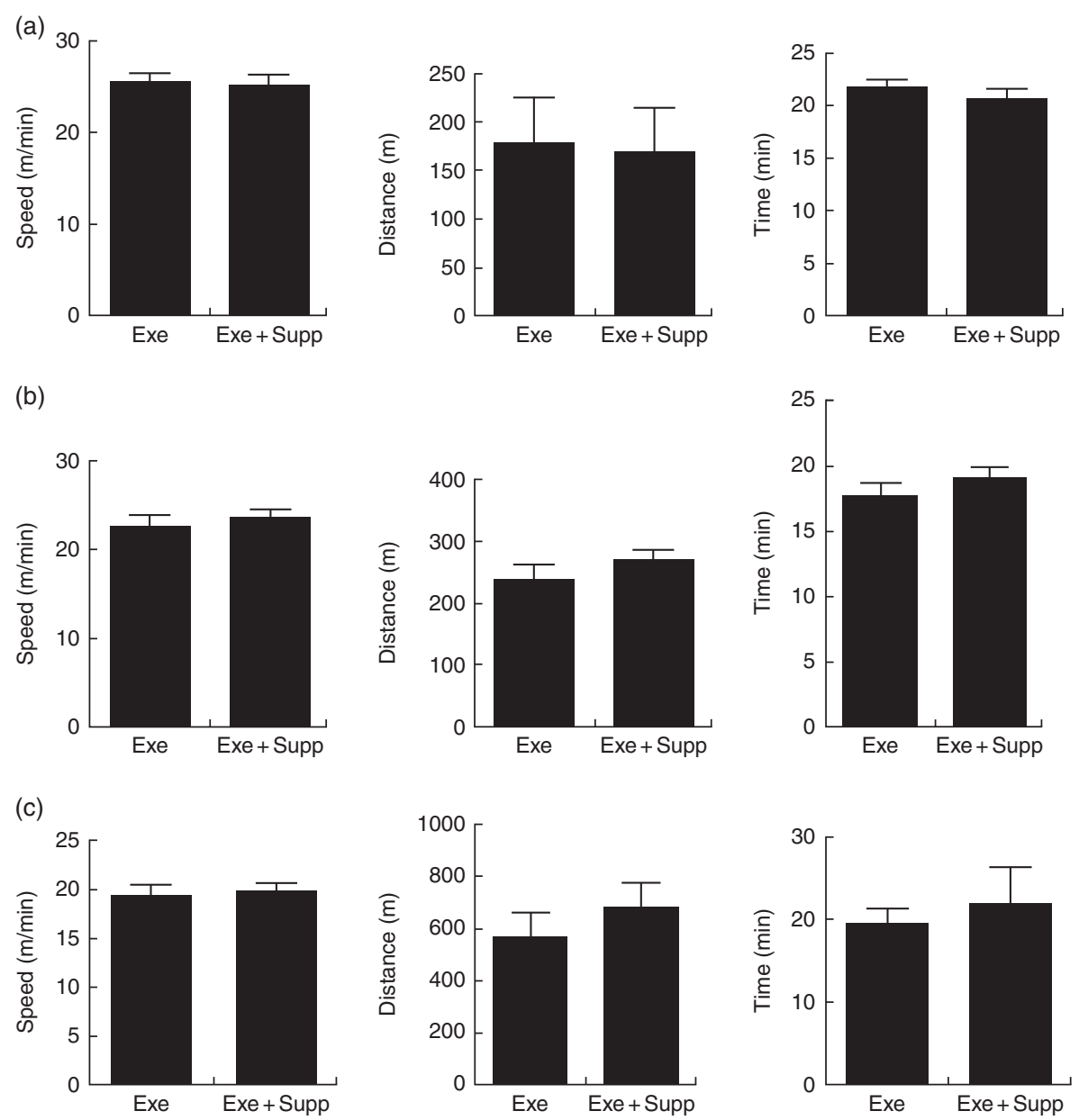

Fig. 1. Animals' performance on treadmill running. (a) Progressive test until exhaustion (maximum test) before treatment (speed in $\mathrm{m} / \mathrm{min}$, distance in $\mathrm{m}$, time in min). (b) Progressive test until exhaustion post treatment (speed in $\mathrm{m} / \mathrm{min}$, distance in $\mathrm{m}$, time in min). (c) Acute exercise at $85 \%$ of the maximum test (speed in $\mathrm{m} / \mathrm{min}$, distance in $\mathrm{m}$, time in min). Six animals from each group were submitted to these tests. Values are averages and standard deviations. No statistical difference was observed between groups. Exe, exercised; Exe + Supp, exercised and supplemented.

other hand, the beverage preserved the original distance between villi, indicating the protector effect of supplementation on high-intensity acute exercise situations $(c=P<0 \cdot 030 v$. Exe).

\section{Immunofluorescence and barrier function protein expression}

Claudin-1 protein labelling is shown in Fig. 4(A), in which it is possible to note that the Supp group showed accumulation of claudin-1 at specific regions (arrows, Fig. 4(A)) and Exe +Supp group presented similar labelling to Ctrl group, whereas the Exe group exhibited a strong labelling reduction. On analysing claudin1 mRNA expression (Fig. 4(B)), we found that supplementation with the fermented milk significantly increased claudin-1 expression (one-way ANOVA: $P<0.001, \mathrm{a}=P=0.004 v$. Ctrl). Intense acute exercise did not change the protein expression in comparison with the Ctrl group. However, the Exe + Supp group presented an increase in claudin- 1 expression $(\mathrm{a}=P=0.004 v$. Ctrl, $\mathrm{b}=P=$ $0.004 v$. Supp, $\mathrm{a}=P=0.005 v$. Ctrl, $\mathrm{b}=P=0.042 v$. Supp and $\mathrm{c}=P=0.004 v$. Exe), showing the influence of supplementation on this protein expression. The ZO-1 labelling pattern was found to be similar to claudin-1, where the Supp group presented increased expression and accumulation in specific epithelial regions (arrows, Fig. 4(C)). The ZO-1 mRNA levels showed similar modulation to claudin-1 (one-way ANOVA: $P=0.008, \mathrm{a}=P=0.017 v$. Ctrl and $\mathrm{c}=P=0.050 v$. Exe), as shown in Fig. 4(D).

\section{Pro-inflammatory cytokine expression}

Cytokine IL-1 $\beta$ expression (Fig. 5(A)) was lower in the group with exercise and fermented milk supplementation (Supp + Exe) (one-way ANOVA: $P<0.05, \mathrm{a}=P<0.018 v$. Ctrl). In the analysis of IL-6 expression (Fig. 5(B)), it was observed that the level of the cytokine in the group with supplementation (Supp) was lower in relation to the control group (Ctrl). The group of exercised rats (Exe) and the group supplemented and submitted to the exercise protocol (Supp + Exe) presented an increased IL-6 compared with the control group. Regarding the analysis of TNF $\alpha$ expression, there was no change in the groups studied.

\section{Antioxidant analyses on blood (serum) and myeloperoxidase activity}

Fig. 6(a) demonstrates antioxidant analyses on blood serum using the FRAP method. No significant differences were found 
(a)
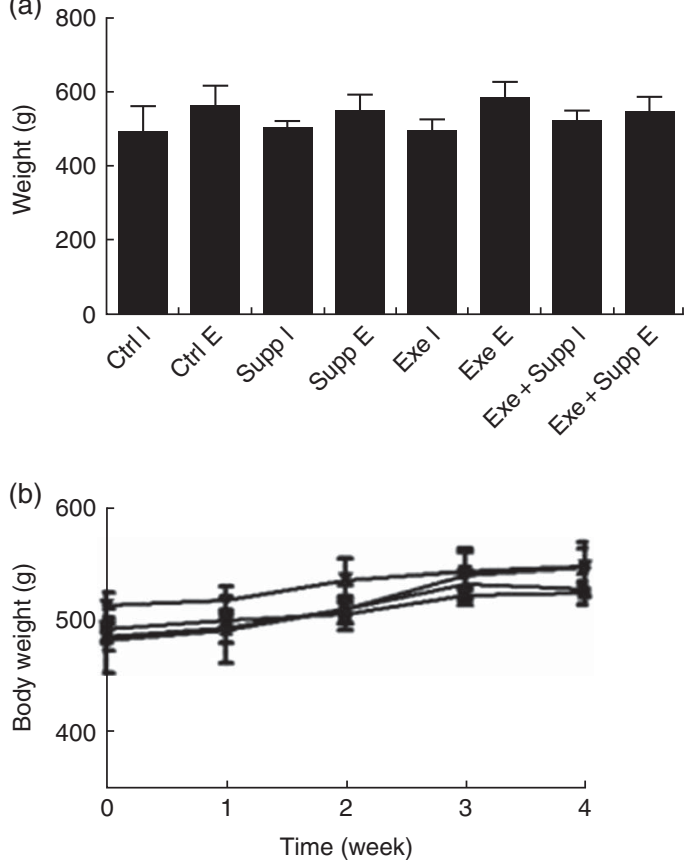

(c)

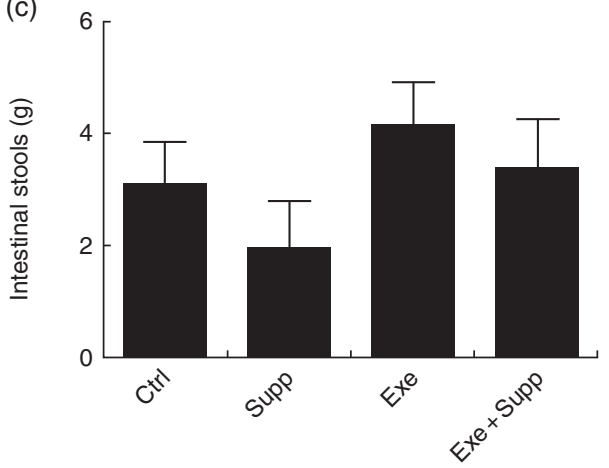

Fig. 2. Body evaluation. (a) Initial weight $v$. final weight. (b) Body weight curve. (c) Faeces weight collected in the colon. Six animals from each group were used for this evaluation. Values are averages and standard deviations. No statistical difference was observed between groups. Ctrl, control; Exe, exercised; Exe + Supp, exercised and supplemented; B: $\longrightarrow$, Ctrl; $\longrightarrow$, Supp; $\longrightarrow$, Exe; $\underset{\longrightarrow}{\longrightarrow}$, Exe + Supp.

between groups. Regarding MPO activity in the distal colon (Fig. 6(b)), an analysis that helps to determine local inflammation, we observed that supplementation seems to decrease neutrophil infiltration, but no statistical difference was observed between groups.

\section{Microbiota analyses by order, genus and species}

In Figs 7-11, we present NGS results of Wistar rats' microbiota. We observed that the order Lactobacillale represented one of the major components of these animals' microbiota, totalising average values of $78 \%$ of total population on control animals (Fig. 7). On comparing Ctrl animals and Supp (not exercised) first, we observed that supplementation with the fermented milk modulated proportions of orders found in the microbiota, increasing the order Bifidobacteriales without decreasing the proportion of Lactobacillale. However, the order Clostridiales was reduced from $21 \%$ to $16 \%$ approximately.

B. animalis did not initially compose rats' microbiota, with $B$. pseudolongum being the only autochthonous belonging to the order Bifidobacteriales, but in reduced proportion (data not shown). The supplementation with the fermented milk introduced B. animalis species in the Exe + Supp and Supp group (data not shown). Intense acute exercise caused a considerable change in the percentage of relative abundance of microbial order on animals' microbiota. Establishing the ratio of Lactobacillale/Clostridiales, we observed that animals from the Ctrl and Supp groups presented values between 4.02 and 4.70 of these two orders; that is, the proportion was four times higher for the Lactobacillale group compared with the Ctrl group and almost five times superior to the supplemented group. This condition was changed by intense exercise, denoting supremacy of Clostridiales (Fig. 8). Supplementation of exercised animals with the fermented milk tended to improve this proportion, elevating Lactobacillale. Nevertheless, there was no statistical difference between groups for the two microbial orders cited.

Taken together, our results obtained for the genus were similar to those obtained for order classification, with reduction of Lactobacillus proportion and increase of Clostridium owing to exercise (Fig. 9). When the Lactobacillus:Clostridium ratio was observed, the same tendency was confirmed, with a statistical difference between the Supp and Exe groups (Fig. 10). To our best knowledge, this result was not previously reported in the literature.

A total of twenty-nine microbial species were identified to comprise Wistar rats' microbiota in this study. To prove the suppressor effect of intense acute exercise on the Lactobacillus genus, we statistically compared the number of sequences of three species found on rats' microbiota, as presented in Fig. 11. We observed a statistically significant reduction of Lactobacillus acidophilus and the tendency of reduction of Lactobacillus murinus $(P=0.07)$. The same figure shows the opposite effect for Clostridium papyrosolvens and Clostridium ruminicantum, which presented a significant increase between animals that suffered stress caused by exercise.

\section{Discussion}

Our results showed that supplementation with fermented milk did not alter the parameters of performance in Wistar rats. The most suitable model for studying sports performance with respect to running is the mouse model. In this study, Wistar rats were used owing to the need for a greater gastric volume to enable daily administration of $2 \mathrm{ml}$ of the beverage in order to reach stipulated amounts of the bioactives compounds. Lollo et $a l{ }^{(32)}$ showed that supplementation of Wistar rats with cheese containing probiotics and submitting them to intense acute exercise prevented immunosuppression caused by strenuous exercise but did not change performance directly. Other research with intense exercise practitioners and endurance athletes showed that supplementation with probiotics also did not alter the physical performance ${ }^{(33-37)}$. A study with humans demonstrated ergogenic effect of supplementation with probiotics, showing a significant increase in race time ${ }^{(38)}$. 

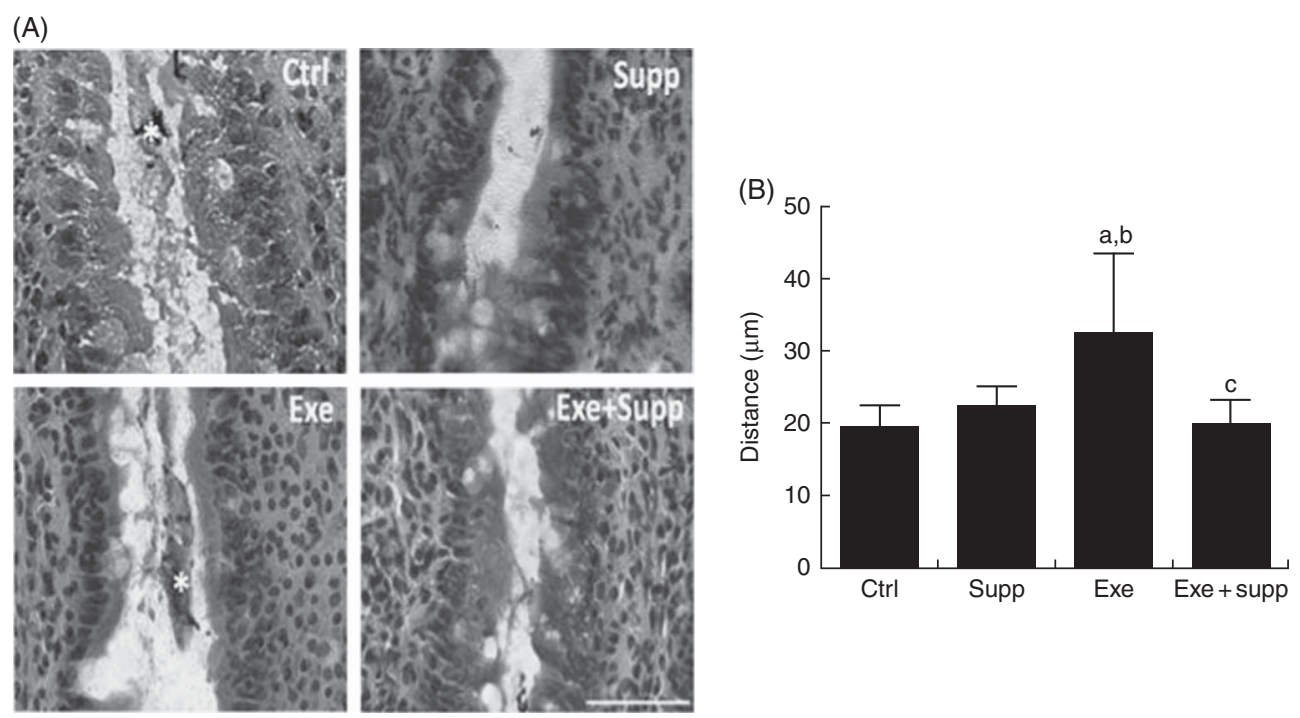

Fig. 3. Histology and intestinal villi distance. (A) Histology from the intestinal colon, bar $=50 \mu m$. (B) Villi distance in the intestinal colon. Six animals from each group were used for this evaluation. One-way ANOVA: $P<0.001$. Values are averages and standard deviations. Ctrl, control; Exe, exercised; Exe + Supp, exercised and supplemented. Post hoc Games-Howell, where $\mathrm{a}=P<0.025$ v. Ctrl, $\mathrm{b}=P<0.098$ v. Supp and $\mathrm{c}=P<0.030$ v. Exe.

In mice, Lactobacillus plantarum TWK10 supplementation improved exercise performance according to Chen et al. ${ }^{(27)}$.

Studies of supplementation with pomegranate extract or pomegranate juice in humans submitted to high-intensity aerobic exercise showed a decrease of oxidative stress caused by exercise but did not show improvement in physical performance $^{(15,39)}$.

Animals supplemented with fermented milk presented lower faecal content in the colon, compared with the other groups. Supporting our results, previous studies have shown that consumption of probiotics decreases intestinal transit time by increasing volume and weight of faeces, gas production, reduction in intestinal $\mathrm{pH}$, increase of SCFA concentration, bacterial metabolism of bile acids and cholecystokinin production ${ }^{(40)}$. In addition, other research also showed that physical exercise could decrease intestinal transit time positively or negatively, as observed in cases of intense exercise where a reduction in intestinal blood flow occurs, causing gastrointestinal discomfort $^{(41-43)}$. Still, in our study, the Exe group without supplementation presented very similar results when compared with the Ctrl group. Van Nieuwenhoven et al. ${ }^{(43)}$ assessed runners and professional bikers regarding intestinal habits, also showing delay in intestinal transit owing to intense exercise.

The intestine is an organ that, beyond digestion and absorption functions, acts as a protective barrier against pathogenic agents ${ }^{(44)}$. Assessing intestinal villi through histology, we observed that the consumption of the fermented milk might favour maintenance of the intestinal physiological structure. Meanwhile, intense acute exercise can negatively alter the intestinal integrity. Tight-junction proteins are intercellular junctions that provide a cell-cell adhesion in enterocytes, playing a critical role in permeability regulation of paracellular barrier $^{(11,12,13,44)}$. In this way, alterations in these proteins lead to the development of pathologies associated with rupture of the intestinal structure and bad nutrient absorption ${ }^{(44-46)}$. Claudins are the most important functional components of tight junctions ${ }^{(44)}$. Regulation of claudins occurs on various levels, such as regulation of transcription, post-translational modification, interaction with cytoplasmic scaffolding proteins and interactions with claudins within the same membrane (cis-interaction), as well as with claudins of neighbouring cells (trans-interaction) $^{(13)}$. For claudins to form functional tightjunction strands, they require additional proteins such as the cytoplasmic scaffold proteins ZO-1 and ZO-2, which bind directly to claudins and link them to the actin cytoskeletal network ${ }^{(47)}$. Intestinal barrier function can be positively modulated by probiotics, according to a review recently published ${ }^{(48)}$. However, the molecular mechanisms behind the effects need to be elucidated. Karczewski et al. ${ }^{(49)}$ administered L. plantarum WCFS1 ( $10^{12}$ cells) in a randomised human crossover study with seven healthy subjects and made biopsies for gene expression analysis and immunohistochemistry. Administration of L. plantarum significantly increased the staining of ZO-1 in the apical part of the cell near the vicinity of the tight junctions, but not the peak fluorescence intensity or cytoplasmic distribution of ZO-1. The mechanism was shown to be dependent on Toll-like receptor 2 signalling. Intense physical exercise can negatively alter intestinal barrier function, as demonstrated by van Wijck et al. ${ }^{(9)}$, whereas consumption of probiotics can favour and re-establish this barrier. Our work demonstrated that supplementation with fermented milk increased tight-junction protein expression. Therefore, probiotics can alter tight-junction protein expression and improve in vitro and animal model barrier function ${ }^{(50,51)}$. Patel et al. ${ }^{(52)}$ administered antibiotics to neonate mice, demonstrating a decrease in barrier function and claudin expression. After administration of Lactobacillus rhamnosus GG, they presented accelerated intestinal barrier maturation and induction of claudin expression. This shows that probiotics administration can accelerate the expression of proteins involved in intestinal barrier function in immature intestine ${ }^{(52)}$. Moreover, Lu et al. ${ }^{(44)}$ have demonstrated that glutamine deprivation decreases 
(A)
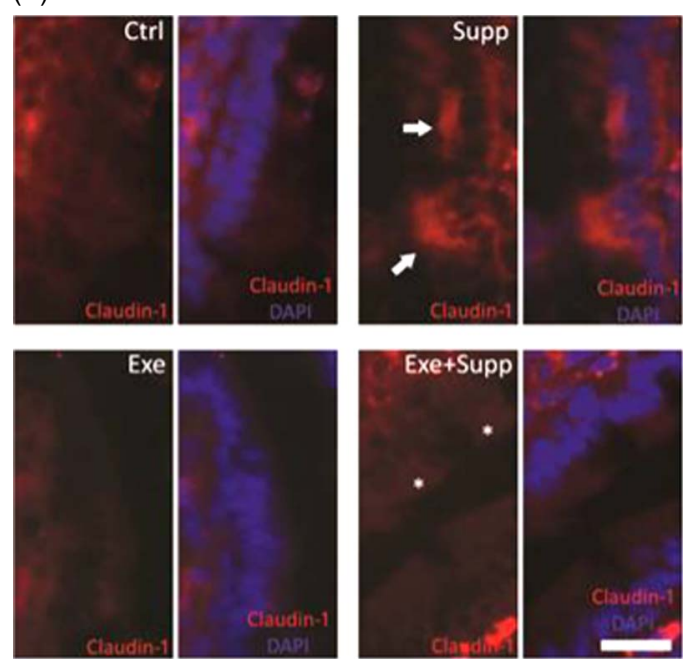

(C)
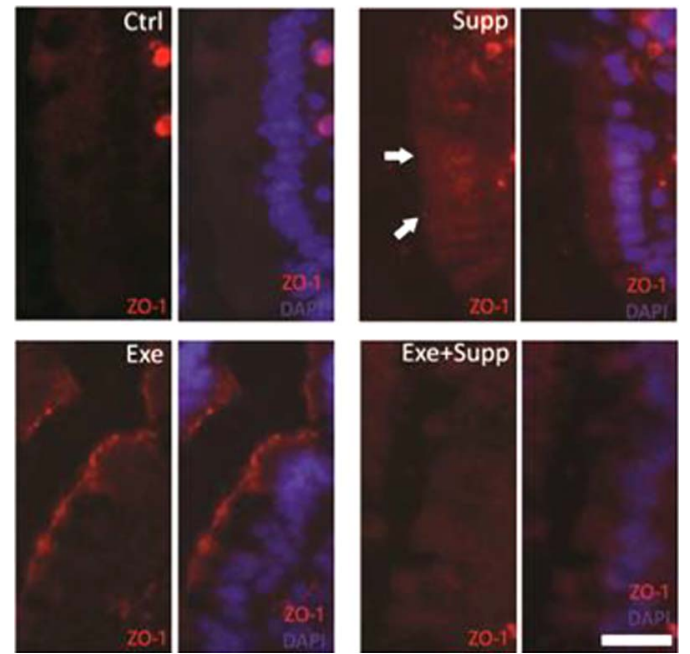

(B)

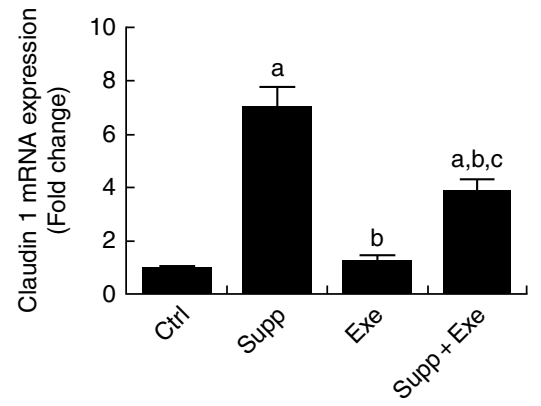

(D)

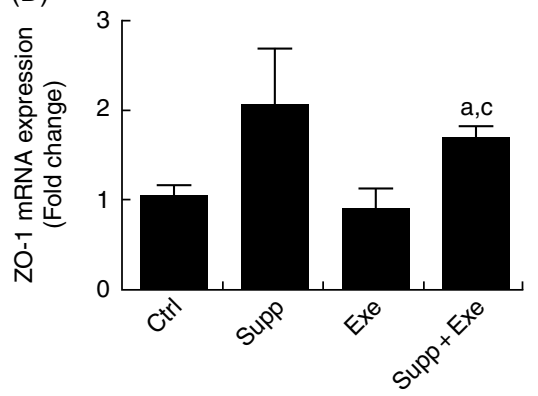

Fig. 4. Immunofluorescence and barrier function protein expression. (A) Claudin-1 immunofluorescence, bar $=50 \mu m$. (B) Claudin-1 expression. One-way ANOVA: $P<0.000$. Values are averages and standard deviations. Post hoc Games-Howell, where $a=P<0.004$ v. Ctrl, $b=P<0.004 v$. Supp, a $=P<0.005$ v. Ctrl, $\mathrm{b}=P<0.042 \mathrm{v}$. Supp, $\mathrm{c}=P<0.004 \mathrm{v}$. Exe. Six animals from each group were used for this evaluation. (C) Zonula occludens-1 (ZO-1) immunofluorescence, $\mathrm{bar}=50 \mu \mathrm{m}$. (D) ZO-1 expression. One-way ANOVA: $P<0.088$. Values are averages and standard deviations. Post hoc Games-Howell, where a $=P<0.017 \mathrm{v}$. Ctrl and $c=P<0.050 v$ v. Exe. Six animals from each group were used for this evaluation. Ctrl, control; Exe, exercised; Exe + Supp, exercised and supplemented.
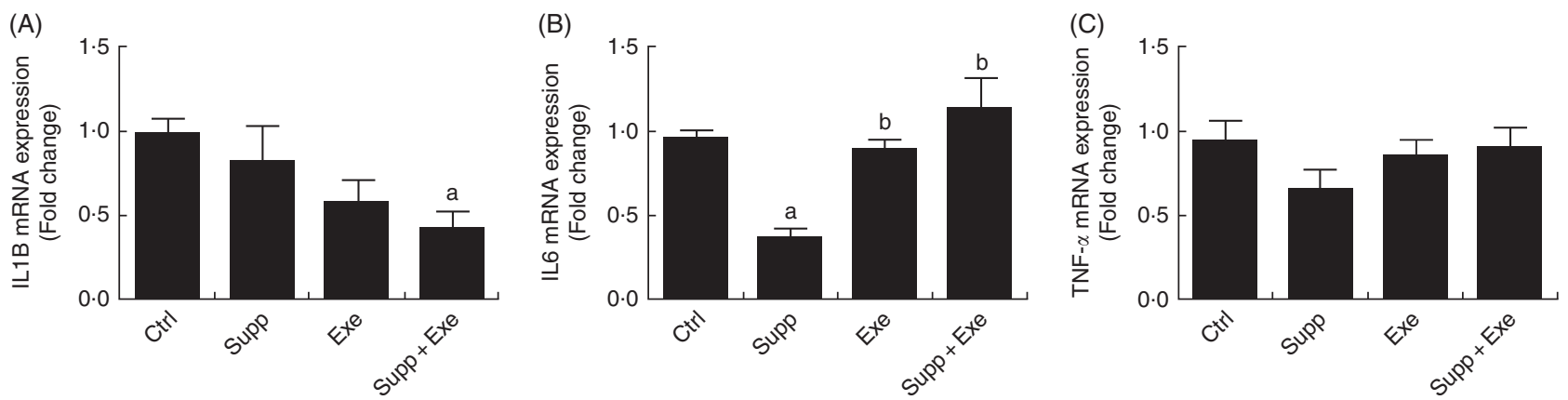

Fig. 5. Pro-inflammatory cytokine expression. (A) IL1B expression. One-way ANOVA: $P<0.051$. Values are averages and standard deviations. Post hoc GamesHowell, where $a=P<0.018 \mathrm{v}$. Ctrl. Six animals from each group were used for this evaluation. (B) IL-6 expression. One-way ANOVA: $P<0.000$. Values are averages and standard deviations. Post hoc Games-Howell, where $a=P<0.000 \mathrm{v}$. Ctrl, $b=P<0.001 \mathrm{v}$. Supp and $b=P<0.008 \mathrm{v}$. Supp. Six animals from each group were used for this evaluation. (C) TNF $a$ expression. One-way ANOVA: $P<0.325$. Values are averages and standard deviations. Six animals from each group were used for this evaluation. Ctrl, control; Exe, exercised; Exe+Supp, exercised and supplemented. 
(a)
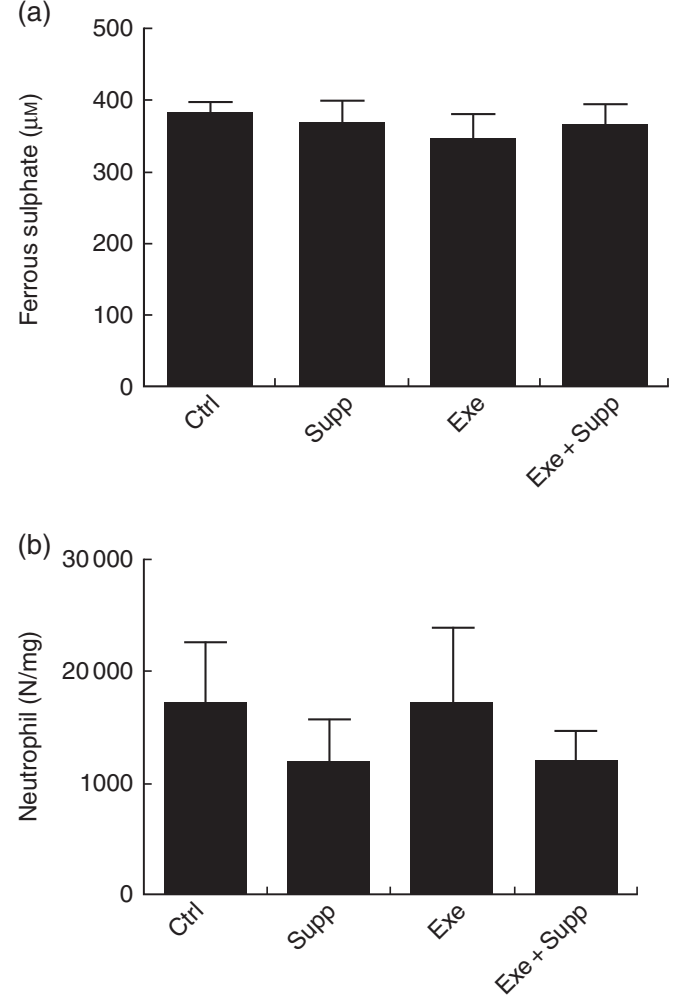

Fig. 6. Antioxidant analysis on blood serum and myeloperoxidase activity. (a) Antioxidant analysis on blood serum by ferric-reducing antioxidant power. (b) Myeloperoxidase activity analysis. Six animals from each group were used for this evaluation. Values are averages and standard deviations. No statistical difference was observed between groups.

claudin-1 expression. The fermented milk administered in this study contains whey protein, specially composed of glutamine, offering a series of benefits, such as strengthening the intestine and immune system and allowing health protection during physical exercise $\mathrm{e}^{(1,53,54)}$.

Cytokines are modulated by the physiological stimulus, such as hormones, nutrients, oxidative stress and physical exercise, and these alterations depend on intensity, duration and type of exercise $^{(1,55-57)}$. Otherwise, on strenuous and intense exercise, circulating levels of pro-inflammatory cytokines TNF- $\alpha$, IL- $1 \beta$ and IL- 6 are increased above normal levels. A previous study showed a 100 -fold increase in plasmatic concentrations of IL-6 and 1 -fold to 2 -fold increase in the concentration of IL-1 $\beta$ and TNF- $\alpha$ after long and intense exercise ${ }^{(58)}$. In the present study, there was no change in IL- $1 \beta$, IL- 6 and TNF $\alpha$ with exercise. Only the rats in the exercise group and those that also received supplementation had reduction in IL- $1 \beta$ cytokine expression.

Cruzat et al. ${ }^{(59)}$ found that supplementation of L-glutamine can attenuate muscle lesion and inflammation, as well as reduce the levels of TNF- $\alpha$ induced by strenuous exercise.

Moreover, our results showed no differences in antioxidant activity on animals' blood serum. However, on assessing MPO activity in the distal colon, we found that supplementation with the functional beverage promotes reduction in neutrophil infiltration, although not statistically significant. A study demonstrated that L-glutamine availability increases neutrophil and lymphocyte activity and function ${ }^{(60)}$. In addition, there is a relationship between neutrophil infiltration, muscle damage and reactive oxygen species generation in the inflammatory process $^{(61)}$. Continuous moderate exercise reduces reactive oxygen species through endogenous antioxidant system adaptation $^{(1,15)}$. Exacerbated inflammation and oxidative stress can cause a decrease in muscle function ${ }^{(15)}$. The endogenous antioxidant system can protect the body against oxidative damage caused by physical exercise. However, they may not be sufficient for elite athletes or in intense exercise ${ }^{(1)}$. Therefore, many strategies have been used to avoid or minimise these damages, such as nutritional interventions with antioxidants ${ }^{(15,18,39)}$. In this context, pomegranate juice is rich in polyphenols such as flavonols, anthocyanins and ellagitannins ${ }^{(15,41)}$, and can be used as an exogenous antioxidant.

Intestine and microbiota are important organs for physical performance, as they are responsible for delivery of nutrients, water and hormones during exercise ${ }^{(7)}$. Intestinal microbiota can neutralise carcinogens and drugs, protect the host from pathogens, stimulate the immune system and modulate intestinal motility ${ }^{(7,62)}$. In addition, alterations in epithelial functions and microbiota composition can exert influence on obesityassociated inflammation ${ }^{(63)}$. The consumption of Lactobacillus and Bifidobacterium can promote beneficial changes in intestinal activity, such as an increase in barrier function ${ }^{(64)}$.

Evaluating animals' microbiota, we observed that the order Lactobacillales was more abundant in Wistar rats from the Ctrl group and that intense acute exercise caused a reduction in this microbial order, that was elevated in these animals and is associated with a well-balanced microbiota (Fig. 7). Animals' supplementation with fermented milk containing pomegranate juice, whey protein and $B$. animalis favoured microbiota dynamic balance in exercised animals, supporting maintenance of relative abundance of Lactobacillus species. We highlight that the probiotic used in our beverage does not belong to the Lactobacillus genus, but to the Bifidobacterium genus. Even so, the supplementation created a favourable microenvironment to re-establish proportions of Lactobacillus suppressed by strenuous exercise. A systematic review from Clark \& $\mathrm{Mach}^{(7)}$ concluded that supplementation with probiotics can result in the release of beneficial metabolites, improving barrier function, immune functions and metabolic functions in athletes ${ }^{(7)}$. B. animalis BB12 was chosen after testing other commercial strains (five Lactobacillus species and two Bifidobacterium species) in preliminary tests This strain achieved better viability results in the fermented milk product developed (data not shown). Moreover, BB12 strain has widespread use in food industry ${ }^{(65)}$ and there are many health benefits reported in the literature ${ }^{(66)}$.

We carefully evaluated the effect of the supplementation on Akkermansia muciniphila proportion, as it has been considered a therapeutic micro-organism with an important role in the mutualism between intestinal microbiota and host, interfering with barrier function and homoeostasis ${ }^{(67)}$. Nevertheless, we did not observe the major proportion of this species related to the supplementation with fermented milk (data not shown), nor did we observe an increase in this genus owing to exercise, as observed by Clarke et $a l^{(68)}$ and Barton et al. ${ }^{(69)}$.

Although there are a few studies on this thematic area so far, some publications point microbiota modulation as one of the 


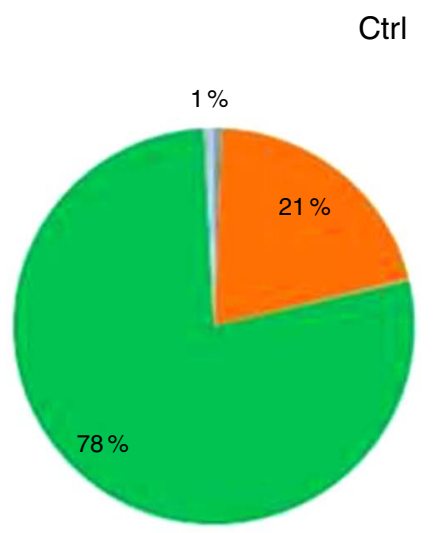

- Bacillales

- Bacteroidales

Bifidobacteriales

n Burkholderiales

= Campylobacterales

= Clostridiales

aggerthellales

Enterobacterales

E Lactobacillales

Methanobacteriales

In Verrucomicrobiales

Supp

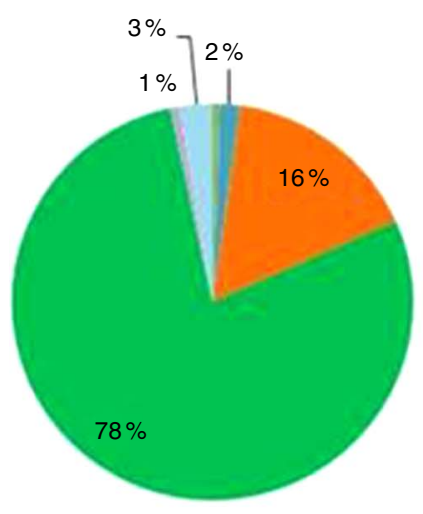

= Bacillales

- Bacteroidales

E Bifidobacteriales

- Burkholderiales

- Campylobacterales

= Clostridiales

* Eggerthellales

Enterobacterales

= Lactobacillales

= Methanobacteriales

- Verrucomicrobiales

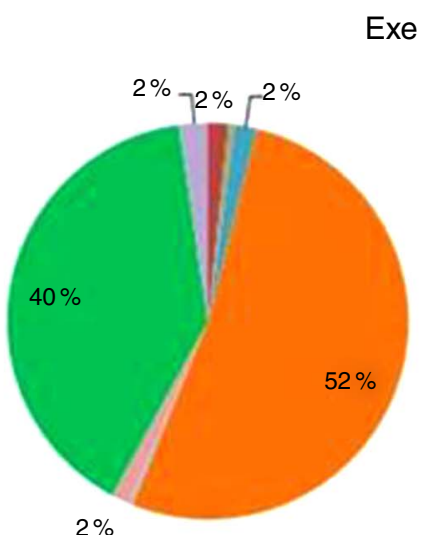

- Bacillales

- Bacteroidales

= Bifidobacteriales

- Burkholderiales

= Campylobacterales

= Clostridiales

E Eggerthellales

- Enterobacterales

- Lactobacillales

nethanobacteriales

IV Verrucomicrobiales

Fig. 7. Microbiota analysis by order. Six animals from each group were used for this evaluation. Values are averages and standard deviations. No statistical difference was observed between groups. Ctrl, control; Exe, exercised; Exe+Supp, exercised and supplemented.

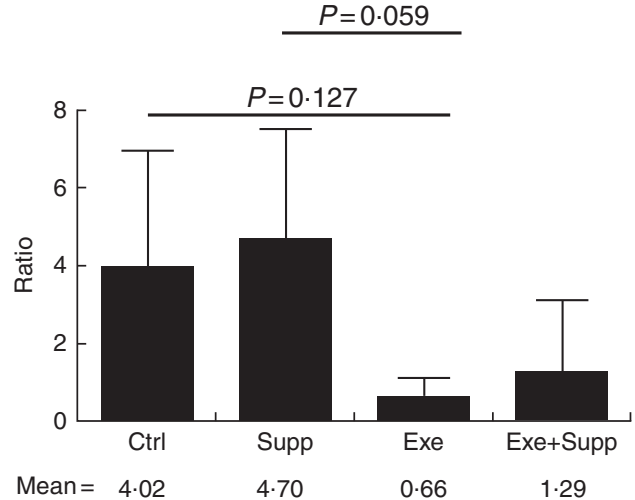

Fig. 8. Ratio of Lactobacillae:Clostridiales orders. Six animals from each group were used for this evaluation. Values are averages and standard deviations. ANOVA test with Games-Howell post-test. Ctrl, control; Exe, exercised; Exe + Supp, exercised and supplemented.

many effects of physical exercises. In the study by Mika et $a l^{(70)}$, young rats submitted to treadmill running presented changes in phylum proportions, with an increase in Bacteroidetes/Firmicutes, a pattern associated with a lean phenotype by some researchers ${ }^{(71,72)}$. On the genus taxonomic level, they observed changes in the proportion of six genera on young and adult rats submitted to running, specifically: Rikenellaceae g_,
Blautia spp., Turicibacter spp., Anaerostipes spp., Methanosphaera spp., Desulfovibrio spp. ${ }^{(70)}$. Our results did not support these findings.

Our research pointed to a statistically significant rise of C. papyrosolvens and C. ruminicantum due to strenuous exercise, as presented in Fig. 11. These findings support results from the study by Batacan et al. ${ }^{(73)}$ on rodents, who observed some species of Clostridium being induced by HIIT exercise (high-intensity interval training), such as Clostridium geopurificans and Clostridium saccharolyticum. It is important to emphasise that the Clostridium genus is associated with dysbiosis, inflammatory bowel diseases and colon cancer ${ }^{(63,74)}$. Interestingly, a study showed that a toxin produced by Clostridium difficile could alter barrier function, modifying tightjunction proteins such as Occludin, $\mathrm{ZO}-1$ and $\mathrm{ZO}-2^{(75)}$.

Another important finding in our study was that strenuous exercise caused a decrease in relative abundance of the Lactobacillus genus (Fig. 9), which was statistically significant for L. acidophilus when compared with control animals (Fig. 11). L. acidophilus is a recognised health promoter species, being widely used by the food and pharmaceutical industry as a probiotic. The supplementation with the fermented milk tended to benefit Lactobacillus proportions and reduced Clostridium proportions (Fig. 9). Sanders suggests that bifidobacteria can modulate colon bacteria activity associated 

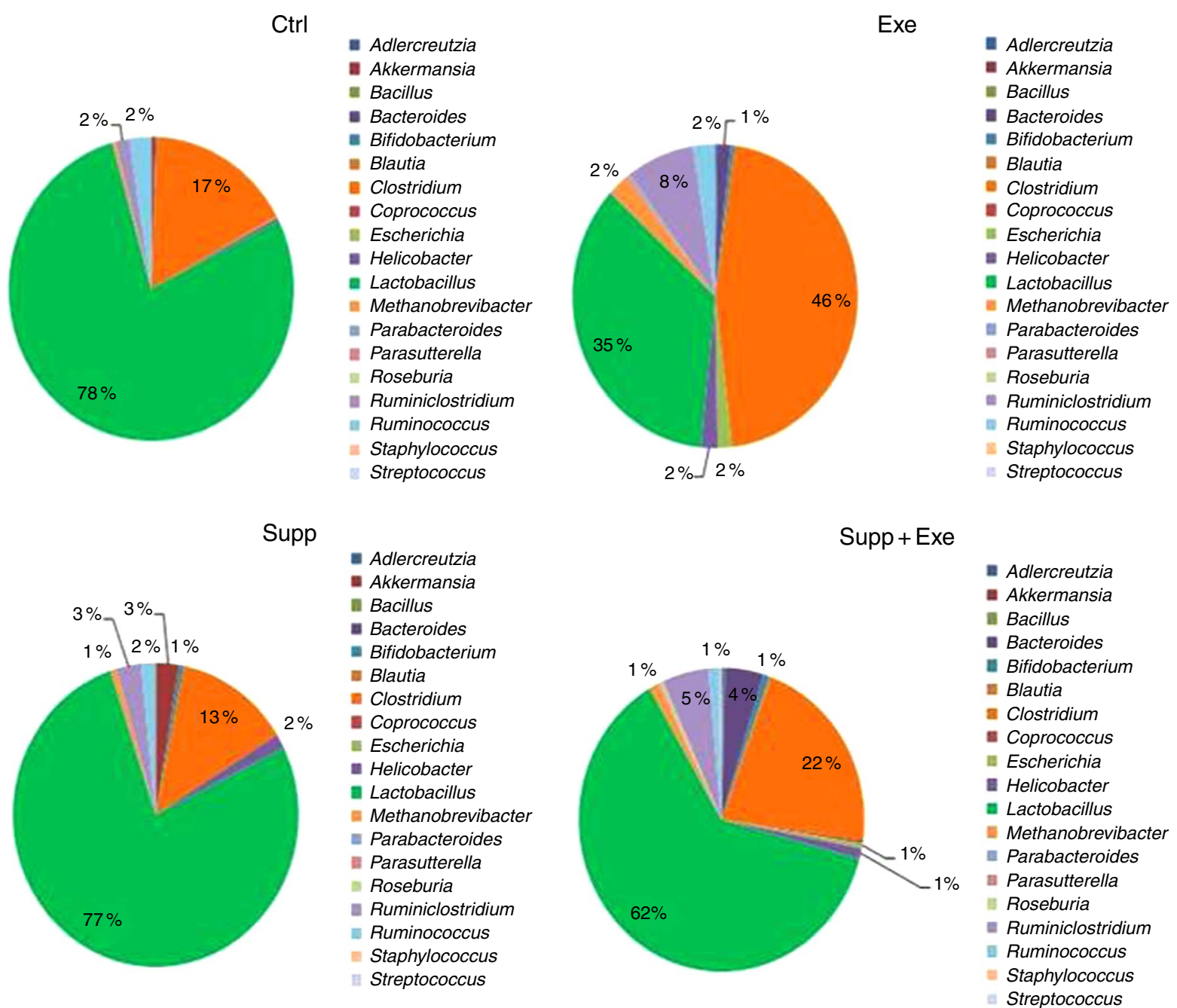

Fig. 9. Microbiota analysis by genus. Six animals from each group were used for this evaluation. Values are averages and standard deviations. No statistical difference was observed between groups. Ctrl, control; Exe, exercised; Exe+Supp, exercised and supplemented.

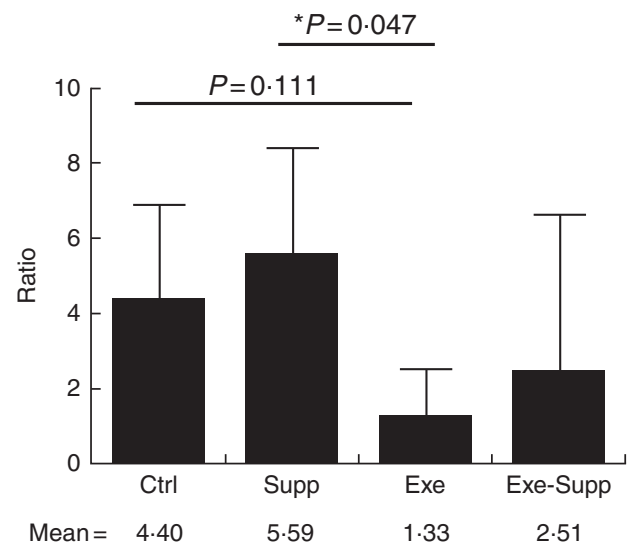

Fig. 10. Ratio of Lactobacillus:Clostridium genera. Six animals from each group were used for this evaluation. Values are averages and standard deviations. ANOVA test with Games-Howell post-test. ${ }^{\star} P<0.05$. Ctrl, control; Exe, exercised; Exe + Supp, exercised and supplemented.

with diseases in animals ${ }^{(76)}$; that is, as bifidobacteria population increases, a reduction is observed for species belonging to the Clostridium genus ${ }^{(63,74)}$.
Literature shows outcomes in different directions regarding Lactobacillus. Choi et al. ${ }^{(77)}$ studied exercised mice, which presented a higher abundance of the order Lactobacillales compared with sedentary animals. Similar results were obtained by Queipo-Ortuno et al. $^{(78)}$, who showed an increase in Lactobacillus proportion owing to exercise, adopting a rat model and treadmill running. Batacan et al. ${ }^{(73)}$ observed an increase of Lactobacillus johnsonii in rodents submitted to light-intensity training running protocol. We suggest that differences in protocols (mainly intensity) are a determining factor to explain the divergence between these findings and the results of our study. In general lines, intense physical exercise seems to compromise intestinal barrier function, decrease intestinal motility and decrease the relative abundance of Lactobacillus species as increase proportion of Clostridium species. L. acidophilus was reduced, and C. papyrosolvens and C. ruminicantum increased by strenuous exercise, with statistically significant differences. It is important to emphasise that the Clostridium genus includes several human pathogens, including Clostridium botulinum (causative agent of botulism) and $C$. difficile, the most significant bacterial cause of diarrhoea in 
Lactobacillus

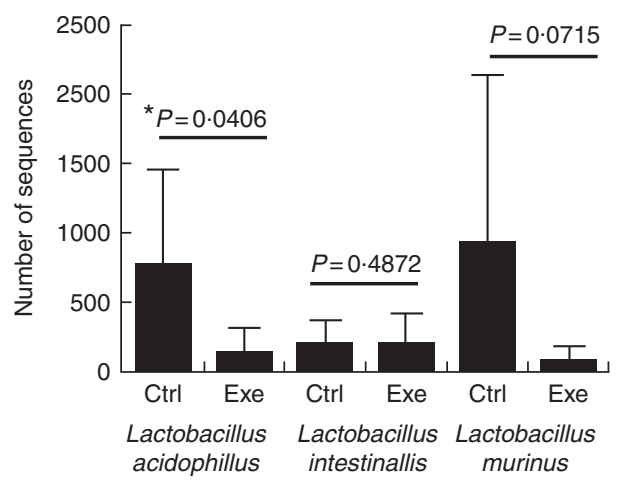

Clostridium

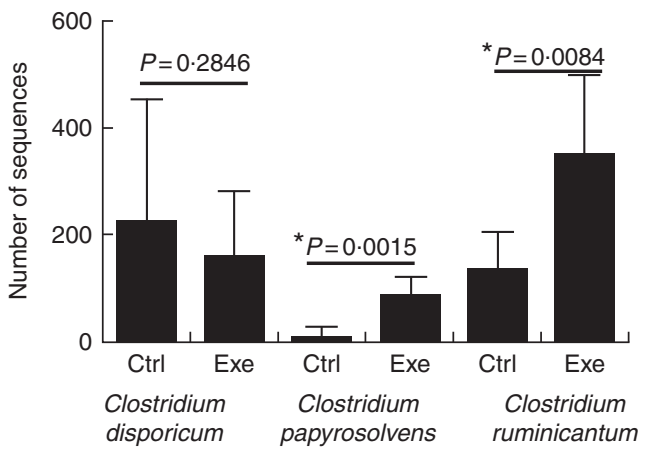

Fig. 11. Exercise decreases Lactobacillus ssp. population and increases Clostridium ssp. population. Six animals from each group were used for this evaluation. Values are averages and standard deviations. Unpaired $t .{ }^{*} P<0.05$. Ctrl, control; Exe, exercised; Exe + Supp, exercised and supplemented.

adults. At present, we observed that strenuous exercise caused microbiota dysbiosis with an increase in the relative proportion of Clostridium ssp., which may represent health risks. Overgrowth of potentially pathogenic micro-organisms can result in tightjunction disruption ${ }^{(79)}$. On the other hand, we observed that the supplementation of the rats with the probiotic fermented milk provided an increase in the structural protein expression of tight junctions, which may result in the strengthening of the barrier function against pathogens. In this way, supplementation proved to be an attractive option in strenuous exercise.

In this study, no statistical difference was obtained in some parameters. The possible factors involved were the variability of the responses associated with the restricted number of animals per group. However, the number of animals used followed the requirements of the Committee on Ethics in Animal Experimentation (CEUA), in accordance with international guidelines.

According to the review published by Clark \& Nuria ${ }^{(7)}$, nowadays, a consensus of probiotic recommendation to supplementation in athletes does not exist. Nevertheless, new studies might point to specific strains or dosages to modulate various adverse events caused by strenuous exercise practice, such as a break of homoeostasis, dysbiosis, endotoxaemia and so on, which might be associated with gastrointestinal discomfort, immunosuppression and even mood changes.

The fermented milk developed in this study was supplemented with probiotics, was rich in protein and polyphenols from pomegranate juice and was capable of benefiting acute exercised animals. Although this beverage was assessed through this prism, its use also can be prospected to humans in acute exercise and in other extreme situations of hypermetabolic states as occurs in malnutrition.

\section{Acknowledgements}

The authors thank the students Luiz Guilherme Salvino da Silva, Vitor Rosetto Muñoz, Rafael Calais Gaspar, Bruna de Melo Aquino, Gabriella Rocha and Franciele Carneiro da Silva for their assistance.

The authors thank Fundação de Apoio à Pesquisa do Estado de São Paulo (FAPESP) for research financing (project no. 15/07299-7) and scholarship concession (no. 15/13972-6). The authors also thank Danisco and Chr-Hansen for kindly providing the probiotic cultures used in this study. The founders FAPESP, Danisco and Chr-Hansen had no role in the design, analysis or writing of this article.

F. M. C.: master's student responsible for all the analyses except for the sequencing of the microbiota. Elaborated the article; I. L. B.: analysis of histology, immunofluorecence and article writing. F. M. S.: molecular biology analyses, interpretation of the results and article writing; P. G. F. Q.: PCRq, RNA extraction and article writing; F. L. P.: analyses of protein quantification, viability of probiotic culture in the fermented milk, phenolic content, animals' supplementation and the protocol of acute exercise; R. M. N. B.: design of the research project, analysis of phenolic content and antioxidant activity of pomegranate juice and blood (serum); J. R. P.: collaborated with acute exercise protocol; D. T. C.: statistical analysis; P. L. C.-F.: collaborated with the design of the project, formulation of the functional beverage and data interpretation; A. E. C. A.: idealisation and coordination of the research project and interpretation of results, especially those related to microbiota analysis.

The authors declare that there are no conflicts of interest.

\section{References}

1. Cruzat VF, Krause M \& Newsholme P (2014) Amino acid supplementation and impact on immune function in the context of exercise. J Int Soc Sports Nutr 11, 61.

2. Newsholme EA \& Parry-Billings M (1990) Properties of glutamine release from muscle and its importance for the immune system. JPEN J Parenter Enteral Nutr 14, 63S-67S.

3. Newsholme P (2001) Why is L-glutamine metabolism important to cells of the immune system in health, postinjury, surgery or infection? J Nutr 131, 2515s-2522s.

4. Newsholme EA \& Calder PC (1997) The proposed role of glutamine in some cells of the immune system and speculative consequences for the whole animal. Nutrition 13, 728-730.

5. Rohde T, MacLean DA \& Pedersen BK (1998) Effect of glutamine supplementation on changes in the immune system induced by repeated exercise. Med Sci Sports Exerc 30, 856-862.

6. Wang B, Wu G, Zhou Z, et al. (2015) Glutamine and intestinal barrier function. Amino Acids 47, 2143-2154.

7. Clark A \& Mach N (2016) Exercise-induced stress behavior, gut-microbiota-brain axis and diet: a systematic review for athletes. J Int Soc Sports Nutr 13, 43. 
8. Nieman DC (2008) Immunonutrition support for athletes. Nutr Rev 66, 310-320.

9. van Wijck K, Lenaerts K, van Loon LJ, et al. (2011) Exerciseinduced splanchnic hypoperfusion results in gut dysfunction in healthy men. PLOS ONE 6, e22366.

10. Lamprecht M \& Frauwallner A (2012) Exercise, intestinal barrier dysfunction and probiotic supplementation. Med Sport Sci 59, 47-56.

11. Garcia-Hernandez V, Quiros M \& Nusrat A (2017) Intestinal epithelial claudins: expression and regulation in homeostasis and inflammation. Ann N Y Acad Sci 1397, 66-79.

12. Fanning AS, Mitic LL \& Anderson JM (1999) Transmembrane proteins in the tight junction barrier. $J$ Am Soc Nephrol 10, $1337-1345$.

13. Gunzel D \& Yu AS (2013) Claudins and the modulation of tight junction permeability. Physiol Rev 93, 525-569.

14. Pyne DB, West NP, Cox AJ, et al. (2015) Probiotics supplementation for athletes - clinical and physiological effects. Eur J Sport Sci 15, 63-72

15. Fuster-Munoz E, Roche E, Funes L, et al. (2016) Effects of pomegranate juice in circulating parameters, cytokines, and oxidative stress markers in endurance-based athletes: A randomized controlled trial. Nutrition 32, 539-545.

16. Gracious Ross R, Selvasubramanian S \& Jayasundar S (2001) Immunomodulatory activity of Punica granatum in rabbits a preliminary study. J Ethnopharmacol 78, 85-87.

17. Varghese S, Joseph MM, RA S, et al. (2017) The inhibitory effect of anti- tumor polysaccharide from Punica granatum on metastasis. Int J Biol Macromol 103, 1000-1010.

18. Sharma P, McClees SF \& Afaq F (2017) Pomegranate for prevention and treatment of cancer: an update. Molecules 22, E177.

19. Drochioiu G, Ciobanu CI, Bancila S, et al. (2016) Ultrasoundbased protein determination in maize seeds. Ultrason Sonochem 29, 93-103.

20. Musci M \& Yao S (2017) Optimization and validation of FolinCiocalteu method for the determination of total polyphenol content of Pu-erh tea. Int J Food Sci Nutr 68, 1-9.

21. Benzie IF \& Strain JJ (1996) The ferric reducing ability of plasma (FRAP) as a measure of 'antioxidant power': the FRAP assay. Anal Biochem 239, 70-76.

22. Witard OC, Jackman SR, Breen L, et al. (2014) Myofibrillar muscle protein synthesis rates subsequent to a meal in response to increasing doses of whey protein at rest and after resistance exercise. Am J Clin Nutr 99, 86-95.

23. Ferreira JC, Rolim NP, Bartholomeu JB, et al. (2007) Maximal lactate steady state in running mice: effect of exercise training. Clin Exp Pharmacol Physiol 34, 760-765.

24. Wang Y \& Qian PY (2009) Conservative fragments in bacterial $16 \mathrm{~S}$ rRNA genes and primer design for $16 \mathrm{~S}$ ribosomal DNA amplicons in metagenomic studies. PLOS ONE 4, e7401.

25. Caporaso JG, Lauber CL, Walters WA, et al. (2011) Global patterns of 16S rRNA diversity at a depth of millions of sequences per sample. Proc Natl Acad Sci US A 108, Suppl. 1, 4516-4522.

26. Takahashi S, Tomita J, Nishioka K, et al. (2014) Development of a prokaryotic universal primer for simultaneous analysis of Bacteria and Archaea using next-generation sequencing. PLOS ONE 9, e105592.

27. Chen YM, Wei L, Chiu YS, et al. (2016) Lactobacillus plantarum TWK10 supplementation improves exercise performance and increases muscle mass in mice. Nutrients $\mathbf{8}, 205$.

28. Games PA, Keselman HJ \& Rogan JC (1983) A review of simultaneous pairwise multiple comparisons. Statistica Neerlandica 37, 53-58.

29. Hill C, Guarner F, Reid G, et al. (2014) Expert consensus document. The International Scientific Association for Probiotics and Prebiotics consensus statement on the scope and appropriate use of the term probiotic. Nat Rev Gastroenterol Hepatol 11, 506-514.

30. Bertazzoni E, Donelli G, Midtvedt T, et al. (2013) Probiotics and clinical effects: is the number what counts? J Chemother 25, 193-212

31. BRASIL. Ministerio da Saude (BRAZIL. Health Ministry) (2016) Alimentos com alegacoes de propriedades funcionais e ou de saude (Foods With Claims of Functional and/or Health Properties). http://portal.anvisa.gov.br/alimentos/alegacoes (accessed January 2018)

32. Lollo PC, Cruz AG, Morato PN, et al. (2012) Probiotic cheese attenuates exercise-induced immune suppression in Wistar rats. J Dairy Sci 95, 3549-3558.

33. Cox AJ, Pyne DB, Saunders PU, et al. (2010) Oral administration of the probiotic Lactobacillus fermentum VRI-003 and mucosal immunity in endurance athletes. Br J Sports Med 44, 222-226.

34. West NP, Horn PL, Pyne DB, et al. (2014) Probiotic supplementation for respiratory and gastrointestinal illness symptoms in healthy physically active individuals. Clin Nutr 33, 581-587.

35. West NP, Pyne DB, Cripps AW, et al. (2011) Lactobacillus fermentum (PCC(R)) supplementation and gastrointestinal and respiratory-tract illness symptoms: a randomised control trial in athletes. Nutr J 10, 30 .

36. Gleeson M, Bishop NC, Oliveira M, et al. (2012) Effects of a Lactobacillus salivarius probiotic intervention on infection, cold symptom duration and severity, and mucosal immunity in endurance athletes. Int J Sport Nutr Exerc Metab 22, 235-242.

37. Valimaki IA, Vuorimaa T, Ahotupa M, et al. (2012) Decreased training volume and increased carbohydrate intake increases oxidized LDL levels. Int J Sports Med 33, 291-296.

38. Shing CM, Peake JM, Lim CL, et al. (2014) Effects of probiotics supplementation on gastrointestinal permeability, inflammation and exercise performance in the heat. Eur J Appl Physiol 114, 93-103.

39. Crum EM, Che Muhamed AM, Barnes M, et al. (2017) The effect of acute pomegranate extract supplementation on oxygen uptake in highly-trained cyclists during high-intensity exercise in a high altitude environment. J Int Soc Sports Nutr 14, 14.

40. Rolfe RD (2000) The role of probiotic cultures in the control of gastrointestinal health. J Nutr 130, 396S-402S

41. Gil MI, Tomas-Barberan FA, Hess-Pierce B, et al. (2000) Antioxidant activity of pomegranate juice and its relationship with phenolic composition and processing. J Agric Food Chem 48, 4581-4589.

42. Simren M (2002) Physical activity and the gastrointestinal tract. Eur J Gastroenterol Hepatol 14, 1053-1056.

43. van Nieuwenhoven MA, Brouns F \& Brummer RJ (2004) Gastrointestinal profile of symptomatic athletes at rest and during physical exercise. Eur J Appl Physiol 91, 429-434.

44. Lu Z, Ding L, Lu Q, et al. (2013) Claudins in intestines: distribution and functional significance in health and diseases. Tissue Barriers 1, e24978.

45. Tokes AM, Kulka J, Paku S, et al. (2005) Claudin-1, -3 and -4 proteins and mRNA expression in benign and malignant breast lesions: a research study. Breast Cancer Res 7, R296-R305.

46. Li Q, Zhang Q, Wang C, et al. (2009) Altered distribution of tight junction proteins after intestinal ischaemia/reperfusion injury in rats. J Cell Mol Med 13, 4061-4076.

47. Overgaard CE, Daugherty BL, Mitchell LA, et al. (2011) Claudins: control of barrier function and regulation in response to oxidant stress. Antioxid Redox Signal 15, 1179-1193.

48. Bron PA, Kleerebezem M, Brummer RJ, et al. (2017) Can probiotics modulate human disease by impacting intestinal barrier function? Br J Nutr 117, 93-107. 
49. Karczewski J, Troost FJ, Konings I, et al. (2010) Regulation of human epithelial tight junction proteins by Lactobacillus plantarum in vivo and protective effects on the epithelial barrier. Am J Physiol Gastrointest Liver Physiol 298, G851-G859.

50. Macpherson AJ \& Harris NL (2004) Interactions between commensal intestinal bacteria and the immune system. Nat Rev Immunol 4, 478-485.

51. Keita AV \& Soderholm JD (2010) The intestinal barrier and its regulation by neuroimmune factors. Neurogastroenterol Motil 22, 718-733.

52. Patel RM, Myers LS, Kurundkar AR, et al. (2012) Probiotic bacteria induce maturation of intestinal claudin 3 expression and barrier function. Am J Pathol 180, 626-635.

53. Castell LM \& Newsholme EA (1997) The effects of oral glutamine supplementation on athletes after prolonged, exhaustive exercise. Nutrition 13, 738-742.

54. Li N \& Neu J (2009) Glutamine deprivation alters intestinal tight junctions via a PI3-K/Akt mediated pathway in Caco2 cells. J Nutr 139, 710-714.

55. Cannon JG (2000) Inflammatory cytokines in nonpathological states. News Physiol Sci 15, 298-303.

56. Nieman DC \& Pedersen BK (1999) Exercise and immune function. Recent developments. Sports Med 27, 73-80.

57. Drenth JP, Van Uum SH, Van Deuren M, et al. (1995) Endurance run increases circulating IL-6 and IL-1ra but downregulates ex vivo TNF-alpha and IL-1 beta production. J Appl Physiol (1985) 79, 1497-1503.

58. Ostrowski K, Rohde T, Asp S, et al. (1999) Pro- and antiinflammatory cytokine balance in strenuous exercise in humans. J Physiol 515, 287-291.

59. Cruzat VF, Rogero MM \& Tirapegui J (2010) Effects of supplementation with free glutamine and the dipeptide alanylglutamine on parameters of muscle damage and inflammation in rats submitted to prolonged exercise. Cell Biochem Funct 28, 24-30.

60. Pithon-Curi TC, Trezena AG, Tavares-Lima W, et al. (2002) Evidence that glutamine is involved in neutrophil function. Cell Biochem Funct 20, 81-86.

61. Niess AM \& Simon P (2007) Response and adaptation of skeletal muscle to exercise-the role of reactive oxygen species. Front Biosci 12, 4826-4838.

62. Nicholson JK, Holmes E, Kinross J, et al. (2012) Host-gut microbiota metabolic interactions. Science 336, 1262-1267.

63. de La Serre CB, Ellis CL, Lee J, et al. (2010) Propensity to highfat diet-induced obesity in rats is associated with changes in the gut microbiota and gut inflammation. Am J Physiol Gastrointest Liver Physiol 299, G440-G448.

64. Mackos AR, Eubank TD, Parry NM, et al. (2013) Probiotic Lactobacillus reuteri attenuates the stressor-enhanced severity of Citrobacter rodentium infection. Infect Immun 81, 3253-3263.

65. Antunes AEC, Silva ERA, Van Dender AGF, et al. (2009) Probiotic buttermilk-like fermented milk product development in a semiindustrial scale: Physicochemical, microbiological and sensory acceptability. Int $J$ Dairy Technol 62, 556-563.

66. Shah NP (2007) Functional cultures and health benefits. Int Dairy J 17, 1262-1277.

67. Everard A, Belzer C, Geurts L, et al. (2013) Cross-talk between Akkermansia muciniphila and intestinal epithelium controls diet-induced obesity. Proc Natl Acad Sci U S A 110, 9066-9071.

68. Clarke SF, Murphy EF, O'Sullivan O, et al. (2014) Exercise and associated dietary extremes impact on gut microbial diversity. Gut 63, 1913-1920.

69. Barton W, Penney NC, Cronin O, et al. (2017) The microbiome of professional athletes differs from that of more sedentary subjects in composition and particularly at the functional metabolic level. Gut 67, 625-633.

70. Mika A, Van Treuren W, Gonzalez A, et al. (2015) Exercise is more effective at altering gut microbial composition and producing stable changes in lean mass in juvenile versus adult male F344 rats. PLOS ONE 10, e0125889.

71. Ley RE, Turnbaugh PJ, Klein S, et al. (2006) Microbial ecology: human gut microbes associated with obesity. Nature $\mathbf{4 4 4}$, 1022-1023.

72. Turnbaugh PJ, Hamady M, Yatsunenko T, et al. (2009) A core gut microbiome in obese and lean twins. Nature $\mathbf{4 5 7}$, 480-484.

73. Batacan RB, Fenning AS, Dalbo VJ, et al. (2017) A gut reaction: the combined influence of exercise and diet on gastrointestinal microbiota in rats. J Appl Microbiol 122, $1627-1638$

74. Hsu CK, Liao JW, Chung YC, et al. (2004) Xylooligosaccharides and fructooligosaccharides affect the intestinal microbiota and precancerous colonic lesion development in rats. $J$ Nutr 134, 1523-1528.

75. Nusrat A, von Eichel-Streiber C, Turner JR, et al. (2001) Clostridium difficile toxins disrupt epithelial barrier function by altering membrane microdomain localization of tight junction proteins. Infect Immun 69, 1329-1336.

76. Sanders ME (1993) Summary of conclusions from a consensus panel of experts on health attributes of lactic cultures: significance to fluid milk products containing cultures. J Dairy Sci 76, 1819-1828.

77. Choi JJ, Eum SY, Rampersaud E, et al. (2013) Exercise attenuates PCB-induced changes in the mouse gut microbiome. Environ Health Perspect 121, 725-730.

78. Queipo-Ortuno MI, Seoane LM, Murri M, et al. (2013) Gut microbiota composition in male rat models under different nutritional status and physical activity and its association with serum leptin and ghrelin levels. PLOS ONE 8, e65465.

79. Hell M, Bernhofer C, Stalzer P, et al. (2013) Probiotics in Clostridium difficile infection: reviewing the need for a multistrain probiotic. Benef Microbes 4, 39-51. 